\title{
REVIEW ARTICLE \\ Capacity to consent to research: The evolution and current concepts
}

\author{
Carmelle Peisah ${ }^{1}$ MBBS (Hons) MD FRANZP, Ute Vollmer-Conna ${ }^{1}$ BSc-Psych (Hon I) PhD \& \\ Scott Y.H. Kim² MD PhD \\ 1 School of Psychiatry, University of New South Wales, Sydney, Australia \\ 2 Department of Psychiatry and Center for Bioethics and Social Sciences in Medicine, University of Michigan, Ann Arbor, MI, USA
}

\author{
Keywords \\ capacity, consent, research

\section{Correspondence} \\ Carmelle Peisah MBBS (Hons) MD FRANZP, \\ Department of Aged Care and Rehabilitation, \\ Bankstown Hospital, 68 Eldridge Road, \\ Bankstown, NSW 2200, Australia. \\ Tel: +61 297228000 \\ Fax: +61298808462 \\ Email: cpeisah62@bigpond.com \\ Received 30 June 2011 \\ Accepted 23 February 2012
}

DOI:10.1111/j.1758-5872.2012.00210.x

\begin{abstract}
The ethical problem of the need to conduct research on the very conditions that impair the ability to consent to such research is widely acknowledged. People with cognitive impairment and mental illness have an equitable right to research being conducted in areas relevant to their treatment and care, and should be given an equitable opportunity to participate in such research, even if they lack capacity. They also have a right to adequate safeguards to protect their interests and respect their wishes in regards to research participation. Provisions for involvement in research of those who are unable to give consent have only developed over the last 50 years. Over this period we have witnessed a proliferation of policies, regulations and laws that govern research involving subjects unable to give consent. There has been a parallel increase in our understanding of the concepts of consent and capacity, specifically as it relates to the research context, with development of standards for research consent and instruments to guide capacity evaluation. We review the evolution of research governance and the underlying ethical principles that underpin such regulations, approaches to capacity evaluation and the use of proxies and advance research directives to facilitate research participation in adults who lack capacity.
\end{abstract}

\section{Introduction}

It is only over the last century that we have recognized that participation in research must be voluntary and informed. Provisions for involvement in research of those who are unable to give consent have only developed over the last 50 years and over this period we have trodden a fine line between ensuring adequate safeguards to protect the interests of those with impaired capacity, while at the same time ensuring research into the very conditions that impair capacity. This paper explores the evolution of research governance and the ethical principles that guide such regulations, as well as approaches to capacity evaluation and research participation in adults who lack capacity.

\section{Evolution of research governance}

The need for informed consent for research was first articulated in 1907 when the British Royal Commission enquired into an experiment in which subjects signed a contract stating the purpose and risks of the experiment, perhaps the first informed consent document (Jonsen et al., 1998a). Subsequently, the Nuremberg Code, in response to the perpetration of the Nazi atrocities involving involuntary, nonconsensual human experimentation, represented the first formal attempt to codify principles to guide human experimentation. The Code deemed essential the voluntary legal consent of the subject, predicated upon an informed disclosure of the nature, duration, purpose, method and hazards of the experiment, 
including the effects on the subject (Jonsen et al., 1998b). Additionally the Code stipulated inclusion of a "revocation of consent" option so that subjects could halt participation at any time if they felt uncomfortable.

The next major attempt to codify research governance was the promulgation of the Declaration of Helsinki in 1964 by the World Medical Association. Hitherto unacknowledged by the Nuremberg Code, a provision for involvement of subjects lacking capacity to participate in research was included in the Declaration. Research was divided into two categories based on the researcher's intent, both categories allowing for consent of a third party (referred to as a "legal guardian") to be substituted for that of incompetent potential participants with compromised autonomy. These categories included:

(i) Therapeutic research - procedures primarily aimed to benefit a particular patient but incidentally also broadened knowledge of the condition or its treatment;

(ii) Non-therapeutic research - where the intent was to extend knowledge to benefit future patients but made no claim to benefit individual patients (World Medical Association, 1964).

This distinction, developed in an attempt to protect vulnerable patients by discouraging their involvement in non-therapeutic and theoretically more hazardous research, has been subsequently abandoned because of the recognition that all research needs to be assessed according to the same criteria. Nevertheless, the distinctive risk-benefit context of research as opposed to treatment and the notion of a risk hierarchy are important considerations in modern research codes.

Over the ensuing 50 years, research regulations have been developed in legislative frameworks and policies of major health governing bodies to implement uniform rules on clinical trials and to protect those who cannot give informed consent. For example, in the USA, the President's Commission for the Study of Ethical Problems in Medicine and Biomedical and Behavioral Research published in 1982 outlined definitions of decision-making capacity as a competent choice which required possession of a set of values and goals, an ability to communicate and to understand information and the ability to reason and to deliberate about one's choices (Kim, 2010).

The current US legislation which deals with informed consent can be found in Title 45 of the Code of Federal Regulations, Part 46 (Department of Health and Human Services, 2005). The legislation has a particular emphasis on a disclosure requirement compris- ing eight criteria, including benefits, alternatives, risks, confidentiality, contact information and compensation, but does not include specific protection for people unable to give consent, beyond the possibility of obtaining permission of the impaired person's surrogate. The regulations refer to "legally authorized representatives" (LAR) with such authority, but the definition of who qualifies as LAR is left to the state and local laws. Because few states have defined who can be LAR, there remains considerable policy uncertainty in the USA. There have been various attempts in the USA, Canada and Europe to propose more comprehensive safeguards, including recommendations for (i) capacity assessment; (ii) proxy decisionmaking and respect for patient assent and dissent, and consistency with patient interests/preferences; (iii) institutional risk-benefit assessment and independent monitors; and (iv) the necessity requirement (i.e. that research must necessarily be performed on subjects who cannot give consent) (Wendler and Prasad, 2001; Resnick et al., 2007). However, no clear resolution or consensus has been reached, particularly in regards to whom such policies apply.

Similarly, in the UK, in 1991, the Medical Research Council published guidelines for Ethical Conduct of Research on the Mentally Incapacitated (http://www.mrc.ac.uk/Utilities/Documentrecord/ index.htm?d=MRC002409), and guidelines for good clinical practice in clinical trials in 1998 (Medical Research Council, 1998). The Medicines for Human Use (Clinical Trials) Regulations 2004 (UK) came into force in the 2004 to cover the conduct of clinical trials on medicinal products. The latter allowed a legal representative of the person not connected with the conduct of the trial to consent to the participation of incompetent adults in medical research (Mason et al., 2006). The Mental Capacity Act 2005 (UK), which came into force in April 2007, governs decisionmaking on behalf of adults who lack mental capacity, both where they lose capacity at some point in their lives, or where the incapacitating condition has been present since birth (http://www.legislation.gov.uk/ ukpga/2005/9/contents).

Australian legislation relating to research involving those who lack capacity to consent was heralded by the Guardianship Act 1987 (NSW), which came into force in August 1989, well in advance of the National Health and Medical Research Council's guidelines and the participation of Australian hospitals and other medical institutions in clinical trials involving those unable to give a valid consent. This early Act only dealt with treatments that were "experimental" (i.e. new treatments that had not yet gained the 
support of a substantial number of medical practitioners or dentists specializing in the area of practice concerned and for which only a tribunal could give consent), rather than clinical trials as such, which were dealt with by subsequent legislation. Queensland legislation authorizing the carrying out of a medical research procedure on an incapable adult followed the NSW Act; and Victoria, while not requiring approval of the Tribunal, provided for a four-step process for authorizing the carrying out of a medical research procedure on an incapable adult. At the time of writing, none of the other Australian states or territories have legislation dealing directly with those who lack capacity to consent to their own treatment receiving experimental treatment or taking part in clinical trials (O'Neill and Peisah, 2011).

Over this period the National Health and Medical Research Council (NHMRC) published guidelines for ethical conduct in human research, which were updated in 2007 (NHMRC, 2007) These guidelines emphasize the entitlement of people with a cognitive impairment, disability or mental illness to participate in research, and the need for care in the research design both to take into account factors that may affect the capacity to receive information, consent and participate, and to make decisions about revoking consent, and to determine if the person's condition makes them susceptible to discomfort or distress. Because of the person's vulnerability, the risks to and burdens imposed on them by the research must be justified by the potential benefits of the research. The NHMRC guidelines regarding consent for people with cognitive impairment, intellectual disability or mental illness comprise the following:

1 Consent must be sought either from the person themselves if they have the capacity to consent, or the person authorized to consent on their behalf;

2 Consent should be witnessed by a person who has the capacity to understand the merits, risks and procedures of the research and is independent of the research team, knows the person and is familiar with their condition;

3 If the cognitive impairment is episodic consent should be sought when the condition does not interfere with the person's capacity;

4 The process of seeking consent should include discussion of any possibility of the person losing their capacity to consent and the person's wishes in that circumstance followed, unless to do so would not be in their best interests;

5 Where consent is sought from a proxy, the researcher should still explain to the participant as far as possible what the research is about
6 Researchers should inform Human Research and Ethics Committees how they propose to determine capacity (including how the decision will be made and by whom, criteria used and process for reviewing capacity during the research)

7 Refusal or reluctance to participate must be respected. Persons with diminished autonomy cannot be made to participate against their expressed wishes at the time (the respect for person principle).

Similarly, over the last five years there has been a proliferation of laws, regulations and guidelines that govern human subject research in several countries in Asia. Clearly, we are not short on laws and regulations guiding human research. In 2011, the Office for Human Research Protections for the US Department of Health and Human Services compiled the International Compilation of Human Research Protection, a list of over 1,000 laws, regulations and guidelines that govern human subject research in 1,010 countries. (www.hhs.gov/ohrp/international/intlcompilation/ hspcompilation-v20101130.pdf)

\section{Ethical considerations}

A number of ethical principles have been entwined in the various codes and legislative frameworks. These ethical concepts are outlined below.

\section{Beneficence}

Beneficence is the requirement to consider the potential benefits (both to society in the form of increased scientific knowledge or new treatments for future patients, and to individual subjects in the form of direct improvement in their health condition) arising from a course of action and balancing these against the potential risks or harm, and also minimizing the risk of harms and burdens. The direct benefit to subjects is akin to the concept of "best interests" which underlies most surrogate decision-making. However, the concept is both highly subjective and has variable meanings depending on the legislative jurisdiction.

\section{Justice}

Justice refers to the need to ensure equitable distribution of resources, including health care and treatment, and to treat all patients equally. People with cognitive impairment and mental illness have an equitable right to research being conducted in areas relevant to their treatment and care, and should be given an equitable opportunity to participate in such research, even if 
they lack capacity (Katonah et al., 2009). Effective treatment of the very conditions which potentially impair capacity is lacking, thus creating the ethical problem of a need for research with those who are incapable of giving their own consent (Kim, 2010; O'Neill and Peisah, 2011). Avoiding this dilemma by limiting research to people who are able to decide for themselves would deprive people who lack capacity of proven therapies for the conditions that specifically affect them (British Medical Association and The Law Society, 2004).

\section{Autonomy}

Autonomy is the act of self-determination or the right to choose, and to do so free from undue influence and coercion. An autonomous decision is usually understood as a competent decision; in the research context this usually means that a person has the capacity to provide informed consent to participate in research. But sometimes facilitating the decision-making of an incompetent person may support a degree of autonomy. It is important to recognize that providing support for decision-making by competent and incompetent patients alike can be achieved without imposing one's will and while still respecting the patient's right to choose. This balance between "coercion" and "abandonment" is all the more important in the context of our often aggressively individualistic, Western ethical frameworks that often impose "mandatory" autonomy (Kim, 2010).

\section{Authenticity}

Although not frequently cited as a principle, authenticity is highly relevant for the context of involving adults in research based on surrogate consent. Authenticity is defined as the congruence between a person's decision and their values, including their beliefs, commitments and relationships (Brudney, 2009). For those with neurodegenerative disorders such as dementia, the values in question are generally those held prior to the development of dementia or at least prior to the development of incompetence. This is also known as the principle of precedent autonomy (Davis, 2002). Although in some settings incongruence with previous decisions and values may signal incompetence, authenticity should not always be seen as a necessary component of competence as a competent person may in fact change their values and commitments over time. In the research context, its importance resides in guiding surrogate decisionmaking (Brudney, 2009; Sulmasy and Snyder, 2010).

\section{Capacity evaluation}

\section{Triggers and thresholds}

The presumption of capacity is the default assumption under Common Law. A "trigger" - usually a behavior, diagnosis, circumstance of event may lead to a questioning of the person's capacity at a point of time (Attorney General's Department of NSW, 2008). In the research context, the decision to challenge this presumption and assess capacity is usually made by the research team developing the research protocol or by the institutional ethics board, and dependent on the research subjects or population. How and who should assess capacity varies according to the protocol and the rigor and degree to which procedures are structured and specified largely depends on the riskbenefit analysis of the research protocol, such that a high-risk study might require an elaborate capacity evaluation assessment scheme with a high capacity threshold performed by independent experienced evaluators (Kim, 2010).

\section{Standards for research consent}

The understanding of capacity to consent to research has evolved from our understanding of the capacity to consent to treatment, which has been derived from American statutory and case law and scholarly sources, and is known as "four abilities" or "four standards" model. The standards as applied to research capacity include:

1 factual understanding of the facts or issues including an understanding of the procedure or treatment its risks and side effects, available options and their advantages and disadvantages and the consequences of participation and non-participation; there may be other elements of informed consent as dictated by the specific jurisdiction.

2 rational manipulation of information or reasoning;

3 appreciation of the nature of the situation as it applies to the subject; and

4 evidencing or communicating a choice, preferably a stable or consistent one (Appelbaum and Roth, 1982; Grisso \& Appelbaum, 1998)

While research capacity is assessed using similar standards to treatment consent, there are extra safeguards (British Medical Association and The Law Society, 2004), with additional "facts" or "issues" that need to be understood, reasoned and appreciated. For example, participants involved in clinical research should understand the difference between treatment and research protocols, including such things as 
randomization and the use of placebos (Resnick et al., 2007).

\section{Instruments}

The use of standard instruments to guide capacity evaluation has been advocated for the purposes of standardization, efficiency, ease of use by research personnel and comparison with published benchmark data to guide capacity thresholds (Kim, 2010).

A number of instruments have been developed to structure and standardize the assessment of capacity. The MacArthur Competence Assessment Tool for Clinical Research (MacCAT-CR) is probably the most widely used instrument for the study of decisionmaking capacity in research. This tool, administered by structured interview which takes approximately 15-20 minutes to complete, comprises 21 items assessing all of the four elements of capacity: understanding, appreciation, reasoning and evidencing a choice (Appelbaum \& Grisso, 2001). The understanding subscale, in particular, has been shown to have reliability and predictive value in identifying patients with mild to moderate Alzheimer's disease who are capable of giving consent (Karlawish et al., 2008a). The MacCAT-CR also had predictive value in determining capacity for informed consent in schizophrenia research (Kim et al., 2007).

Since the development of the MacCAT-CR there have been attempts to develop more parsimonious but still valid and reliable tests for capacity to consent to research participation, particularly for cognitively impaired individuals who may have limited ability to concentrate as a result of both physical and cognitive problems. A number of shorter tools have been developed, their brevity limiting their assessment to one or two components of the capacity construct, and thus their role to screening rather than capacity determination (Saks et al., 2002; Resnick et al., 2007).

Differentiating capable from incapable subjects remains an issue, despite the aid of these standardized tools as they yield dimension scores but not categorical outcomes (Kim et al., 2001). The currently identified goal of research in this area is to find markers of potential impaired capacity. Ideally they are sensitive, brief questionnaires targeting key aspects of disclosed information, which effectively screen for participants warranting more comprehensive capacity evaluations (Palmer and Jeste, 2006). In many research centers, the assessment of decisional capacity, particularly of subjects with dementia, lacks uniformity, when it is performed at all. Where decisional capacity is either formally or informally assessed, a variety of methods are used ranging from clinical judgment to extrapolation from psychometric performance (Karlawish et al., 2002) A study of clinicians' perceptions of videotaped capacity interviews involving two scenarios of different risk (i.e. a medication-randomized clinical trial and a neurosurgical clinical trial), showed that although clinicians used a risk-sensitive model of capacity determination (a higher degree of capacity required in higher-risk situations), there is considerable unexplained variability in their judgments, most likely due to variations in threshold between capacity assessors (Kim et al., 2006; S.Y.H. Kim et al., 2011).

\section{Capacity and diagnosis: Patient predictors of capacity}

Considerable discussion still surrounds issues related to the capacities of patients with neuropsychiatric disorders to consent to research. It is agreed, however, that age and diagnosis - indeed any measure not specifically based on measuring functional abilities to make decisions - should not be viewed as determinants of decisional capacity (Palmer and Jeste, 2006). The presence of disorder does not imply impairment in capacity. Although some studies have found, for example, that even relatively mild Alzheimer's disease significantly impairs consent-giving capacity (Kim et al., 2006) there is considerable heterogeneity in decision-making capacity even within each diagnostic group, particularly amongst those with schizophrenia, sufficient to warrant individualized consideration of capacity (Jeste et al., 2006; Palmer and Jeste, 2006). Of course, this will clearly depend on how capacity is defined or tested, and on the complexity of the capacity task. Even mild cognitive impairment (MCI) can affect decisional capacity for complex research protocols (Buckles et al., 2003; Jefferson et al., 2008).

Another important factor is the need to incorporate the overall risk-benefit profile of the research protocol into the final categorical determinations of capacity. Studies have shown that consultation psychiatrists do follow the often-recommended (National Bioethics Advisory Comission, 1998) guidance that as risks increase, the threshold for competence should increase also (Kim et al., 2006; S. Kim et al., 2011).

Individual consideration of capacity is equally important for those with mental illnesses such as mood disorder or schizophrenia. Although the presence of bipolar disorder appears to be a risk factor for impaired understanding of information disclosed under standard consent procedures, the diagnosis should not be equated with a lack of competence to consent (Palmer et al., 2007). 
In studies of patients with bipolar disorder and schizophrenia, although the strongest correlates of capacity (particularly, understanding and appreciation of disclosed information) were cognitive test scores, there was little evidence of differential relationships between individual cognitive abilities on neuropsychological testing and specific dimensions of capacity. Understanding was correlated with severity of negative symptoms and of general psychopathology (Jeste et al., 2006; Palmer et al., 2007).

\section{Research involving adults who have cognitive impairment}

It has been suggested that as a rule of thumb, people who lack capacity to consent for themselves should only ever be involved in projects from which they, or others with similar conditions are likely to benefit, and which cannot be undertaken otherwise on people who are able to consent (British Medical Association and the Law Society, 2004). In two Australian states (New South Wales and Queensland), legislation requires that the state's administration and guardianship tribunal must be satisfied, before they approve of a trial involving those unable to give consent, that, having regard to the potential benefits and risk of participation in the trial, it is in the best interests of the patients who suffer from the relevant condition that they take part in the trial (see Guardianship Act, 1987 [NSW]). To meet this criterion, the Tribunal has to be presented with some evidence that taking part in the clinical trial will be of benefit to the class of incapable participants themselves. The evidence has to go beyond the suggestion that, if they had capacity, they would feel good about themselves taking part in the trial.

Beyond legislative requirements of ensuring a best interests standard, or a risk-benefit analysis, there are number of ways of supporting the involvement of incapacitated subjects in research. The first is by facilitating their own consent. The second is by considering their previously expressed opinions as there is some scope for including those who have made advance directives dealing with the question of medical research. The third is by obtaining the consent of proxy decision-makers.

\section{Facilitating the consent process and involvement of cognitively impaired subjects}

Decisional capacity is not necessarily an unmodifiable trait (Jeste et al., 2003). A variety of interventions or "remediations" can enhance understanding of informed consent for research. Capacity may be enhanced by providing information in an easily understandable form. Even among individuals with psychiatric illness or cognitive impairment, deficits in understanding can be remedied with certain educational interventions. Deficits in patients' understanding of informed consent may be related partially to poorly conceived, written, or organized consent materials. Effective interventions include corrected feedback, multiple learning trials, and more organized or simplified consent forms (Dunn and Jeste, 2001). For example, an interactive dialogue between patient and investigator with clarification of key elements in the consent form and repeated presentation of information is likely to aid understanding of disclosed information among patients with schizophrenia (Jeste et al., 2003, 2006).

The wording of questions in the consent process can affect the responses of the participant. For example, in a study of 102 middle-aged and older outpatients with schizophrenia or related psychotic disorders and 20 normal comparison subjects, patients had more difficulty than normal comparison subjects on open-ended questions, including those asking about study procedures, time involved, and potential risks and benefits. Among patients, the enhanced procedure was associated with better performance on questions about potential risks and time required than the routine procedure. It is these "problem areas" in the understanding of informed consent that should be the focus of attempts to improve the consent process for patient participants with severe mental illness (Dunn and Jeste, 2003).

\section{Advance research directives}

There have been proposals for research regulations and guidelines in some states of the USA and Canada to require that research involving cognitively impaired adults be limited to those who have completed a formal, documented advance research directive while competent (Wendler and Prasad, 2001; Muthappan et al., 2005). Yet, this requirement may impede the carrying out of important research. One study from the USA found that only $11 \%$ of adult inpatients had completed an advance research directive, even when given the opportunity to do so in a clinical research setting. Of those who completed research directives, $76 \%$ were willing to participate in research that might help them, 49\% were willing to participate in research that would not help them and posed minimal risk, and $9 \%$ were willing to participate in research that would 
not help them and posed greater than minimal risk. The authors suggested more flexible approaches to protect these individuals such as:

1 developing advance directives that address both research and clinical care;

2 requiring advance research directives for subjects at high risk for losing the ability to consent, such as individuals with mild Alzheimer's disease enrolling in longitudinal studies; and

3 developing mechanisms to allow individuals to reject future research participation, while making them aware that formally documenting a preference to decline future research may preclude them from all research including research with the potential to benefit them (Muthappan et al., 2005).

\section{Proxy consent}

Research in the UK and the USA shows that future research involving incapacitated older patients will need to rely heavily on proxy or substitute consent provision (Mason et al., 2006). Again, the role of substitute decision-makers varies enormously from jurisdiction to jurisdiction in terms of who may serve as surrogate (in Australia: "persons responsible", guardians, statutory health attorneys or, in one state doctors when the persons responsible cannot be contacted or ascertained), the types of research the surrogate may consent to in terms of risk and benefits, and the matters they must take into account before giving that consent. Such matters include:

1 the current views of the incapable person must be taken into consideration;

2 the past views must be taken into consideration, if they can be ascertained; and

3 the welfare and interests of that person must be given paramount consideration. In general, most jurisdictions have attempted to provide some limits on the risks borne by the subjects who are entered into research studies via surrogate permission.

Over the last few years there has been a burgeoning interest in the appointment of proxies for research consent as alternatives to making advance research directives (Stocking et al., 2006). It is thought that persons suffering from neurodegenerative disorders such as dementia may retain significant abilities, including sufficient capacity for delegating one's authority for giving consent to research, even if they are not capable of giving independent consent themselves (Kim and Appelbaum, 2006; Kim and Kieburtz, 2006; Kim et al., 2011).

As with any situation of proxy consent, in the absence of an advance directive, surrogates have to make research decisions based on informal evidence of the person's preferences. Yet, consent decisions of legal representatives may not necessarily reflect those of patients themselves and may result in underrecruitment of patients, who if given the choice themselves may have chosen to participate. From 2,445 potentially eligible but incapacitated patients, proxy consent from a relative led to trial participation of only $3.6 \%$ patients. The reasons attributed to this were that a large number of incapacitated patients had no relative available for consent $(2,286)$, but also $45 \%$ of relatives approached refused to provide consent compared with $18 \%$ of patients who refused to participate in the trial. Proxy consent allowed only a small increase in trial recruitment of incapacitated patients. The authors suggested that the finding that more relatives than patients refused to consent implies either more caution amongst proxies than the patients themselves, or that proxies used different criteria when making their decision (Mason et al., 2006). Such caution amongst proxies may reflect a natural protective posture as well as a lack of willingness to take on too much responsibility, a beneficent or best interests position that provides a protective function for vulnerable subjects who lack capacity.

Similarly, Stocking et al. (2006) interviewed 149 dyads of dementia patients and family proxies about future enrollment in five types of research and while they found that patients chose to cede future decisionmaking to their proxies in $82.9 \%$ of the trials, patients ceded decisions to their proxies in $80.7 \%$ of those trials about which the dyad had given opposite answers. Such a mismatch between patient and proxy views regarding enrolment in research studies is more likely to occur when proxies use a best interest rather than a substituted judgment standard for decisionmaking. Karlawish et al. (2008b) examined the views of Alzheimer's disease patients and their proxies at 13 research sites in the USA and found that proxies made research enrollment decisions based on what they thought would maximize the patient's well-being as opposed to a substituted judgment standard. Reasons proxies give for participating in research include hope of direct or indirect benefits to the patient, caregiver, or patient's descendants; desperation; trust in the investigator; belief in the goodness of research; and altruism, although these reasons vary according to the type of research, such that in drug trials hope of direct benefit prevails while in studies not evaluating a potential therapy more altruistic concerns predominate (Sugarman et al., 2001).

Interestingly, there is evidence that some people value the future role of surrogates more than their 
currently held preferences and are thus willing to cede future decisions to participate in research that they currently do not wish to participate in to their surrogates (Kim et al., 2009). Conversely, it must be recognized that a sizeable minority do not wish to do so (Stocking et al., 2006).

\section{Conclusion}

It is only since the 1960s that research policies have made provision for involvement of subjects lacking capacity to participate in research. However, over this short period we have witnessed an exponential growth internationally of policies, regulations and laws that govern research involving subjects unable to give consent. Shaped by fundamental ethical principles of autonomy, justice, beneficence and authenticity, as well as our growing understanding of the concepts of consent, capacity, and surrogate decisionmaking, such policies vary from jurisdiction to jurisdiction according to the different emphasis placed on each of these constructs. Internationally, we are not short on guidelines, but rather, we are short on consensus.

\section{References}

Appelbaum P.S., Grisso T. (2001) The MacArthur Competence Assessment Tool for Clinical Research (Mac CAT-CR). Professional Resource Press, Sarasota, FL.

Appelbaum P.S., Roth L.H. (1982) Competency to consent to research. Arch Gen Psychiatry. 39, 951-958.

Attorney General's Department of NSW (2008) Capacity Toolkit Diversity Services Unit, ecoDesign ecoPrint, Wolli Creek.

British Medical Association, The Law Society (2004) Assessment of Mental Capacity - Guidance for Doctors and Lawyers, 2nd edn. BMJ Books, London, p. 137.

Brudney D. (2009) Choosing for another: beyond autonomy and best interests. Hastings Cent Rep. 39, 31-37.

Buckles V.D., Powlishta K.K., Palmer J.L., et al. (2003) Understanding of informed consent by demented individuals. Neurology. 61, 1662-1666.

Davis J.K. (2002) The concept of precedent autonomy. Bioethics. 16, 114-133.
Department of Health and Human Services (2005) Code of Federal Regulations Title 45: Public Welfare, Part 46: Protection of Human Subjects.

Dunn L.B., Jeste D.V. (2001) Enhancing informed consent for research and treatment. Neuropsychopharmacology. 24, 595-607.

Dunn L.B., Jeste D.V. (2003) Problem areas in the understanding of informed consent for research: Study of middle-aged and older patients with psychotic disorders. Psychopharmacology (Berl). 171, 81-85.

Grisso T., Appelbaum P.S. (1998) Assessing Competence to Consent to Treatment: A Guide for Physicians and Other Health Professionals. Oxford University Press, New York.

Guardianship Act (1987) (NSW) s 45(2)(d). See 16. 3. 1.

Jefferson A.L., Lambe S., Moser D.J., Byerly L.K., Ozonoff A., Karlawish J.H. (2008) Decisional capacity for research participation in individuals with mild cognitive impairment. J Am Geriatr Soc. 56, 1236-1243.

Jeste D.V., Dunn L.B., Palmer B.W., et al. (2003) A collaborative model for research on decisional capacity and informed consent in older patients with schizophrenia: bioethics unit of a geriatric psychiatry intervention research center. Psychopharmacology (Berl). 171, 68-74.

Jeste D.V., Depp C.A., Palmer B.W. (2006) Magnitude of impairment in decisional capacity in people with schizophrenia compared to normal subjects: an overview. Schizophr Bull. 32, 121-128.

Jonsen A.R., Veatch R.M., Walters L. (1998a) The ethics of research with human subjects: a short history. In: Source Book in Bioethics. Georgetown University Press, Washington, DC, pp. 5-10.

Jonsen A.R., Veatch R.M., Walters L. (1998b) Source Book in Bioethics: A Documentary History. Georgetown University Press, Washington, DC, pp. 11-12.

Karlawish J., Kim S.Y.H., Knopman D., van Dyck C.H., James B.D., Marson D. (2008a) Interpreting the clinical significance of capacity scores for informed consent in Alzheimer disease clinical trials. Am J Geriatr Psychiatry. 16, 568-574.

Karlawish J., Kim S.Y.H., Knopman D., van Dyck C.H., James B.D., Marson D. (2008b) Views of Alzheimer disease patients and their study partners on proxy consent for clinical trial enrollment. Am J Geriatr Psychiatry. 16, 240-247.

Karlawish J.H.T., Knopman D., Clark C.M., et al. (2002) Informed consent for Alzheimer's disease clinical trials: a survey of clinical investigators. IRB. $24,1-5$. 
Kim S., Karlawish J.H., Kim H.M., Wall I.F., Bozoki A.C., Appelbaum P.S. (2011) Preservation of the capacity to appoint a proxy decision maker: implications for dementia research. Arch Gen Psychiatry. 68, 214-219.

Kim S.Y., Appelbaum P.S., Swan J., et al. (2007) Determining when impairment constitutes incapacity for informed consent in schizophrenia research. Br J Psychiatry. 191, 38-43.

Kim S.Y.H. (2010) Evaluation of Capacity to Consent to Treatment and Research. Oxford University Press, New York.

Kim S.Y.H., Appelbaum P.S. (2006) The capacity to appoint a proxy and the possibility of concurrent proxy directives. Behav Sci Law. 24, 469-478.

Kim S.Y.H., Kieburtz K. (2006) Appointing a proxy for research consent after one develops dementia; the need for further study. Neurology. 66, 1298-1299.

Kim S.Y.H., Caine E.D., Currier G.W., Leibovici A., Ryan J.M. (2001) Assessing the competence of persons with Alzheimer's disease in providing informed consent for participation in research. Am J Psychiatry. 158, 712-717.

Kim S.Y.H., Caine E.D., Swan J.G., Appelbaum P.S. (2006) Do clinicians follow a risk-sensitive model of capacity-determination? An experimental video survey. Psychosomatics. 47, 325-329.

Kim S.Y.H., Kim H.M., Langa K.M., Karlawish J.H., Knopman D.S., Appelbaum P.S. (2009) Surrogate consent for dementia research: a national survey of older Americans. Neurology. 72, 149-155.

Kim S.Y.H., Appelbaum P.S., Kim H.M., et al. (2011) Variability of judgments of capacity: experience of capacity evaluators in a study of research consent capacity. Psychosomatics. 52, 346-353. doi:10.1016/j.psym.2011.01.012.

Mason S., Barrow H., Phillips A., et al. (2006) Brief report on the experience of using proxy consent for incapacitated adults. J Med Ethics. 32, 61-62.

Medical Research Council (1998) MRC Guidelines for Good Clinical Practice in Clinical Trials. MRC, London.

Medical Research Council (2007) [Cited 1 Jun 2011.] Available from URL: http://www.mrc.ac.uk/Utilities/ Documentrecord/index.htm?d=MRC002409

Muthappan P., Forster H., Wendler D. (2005) Research advance directives: protection or obstacle? Am J Psychiatry. 162, 2389-2391.
National Bioethics Advisory, C. (1998) Research Involving Persons with Mental Disorders That May Affect Decisionmaking Capacity. NBAC, Rockville, MD.

NHMRC (National Health and Medical Research Council), Australian Research Council (2007) Australian Vice-Chancellors' Committee. National Statement on Ethical Conduct in Human Research, Australian Government, Canberra, p65-66.

O'Neill N., Peisah C. (2011) Capacity and the Law. SydUPLawbkl. Sydney University Press. (Online Publication). [Cited 1 November 2011 .] Available from URL: http://www.austlii.edu.au/au/journals/ SydUPLawBk/2011/1.html

Palmer B.W., Jeste D.V. (2006) Relationship of individual cognitive abilities to specific components of decisional capacity among middle-aged and older patients with schizophrenia. Schizophr Bull. 32, 98-106.

Palmer B.W., Dunn L.B., Depp C.A., Eyler L.T., Jeste D.V. (2007) Decisional capacity to consent to research among patients with bipolar disorder: comparison with schizophrenia patients and healthy subjects. J Clin Psychiatry. 68, 689-696.

Resnick B., Gruber-Baldini A.L., Pretzer-Aboff I., et al. (2007) Reliability and validity of the evaluation to sign consent measure. Gerontologist. 47, 69-77.

Saks E.R., Dunn L.B., Marshall B.J., Nayak G.V., Golshan S., Jeste D.V. (2002) The California Scale of Appreciation: a new instrument to measure the appreciation component of capacity to consent to research. Am J Geriatr Psychiatry. 10, 166-174.

Stocking C.B., Hougham G.W., Danner D.D., Patterson M.B., Whitehouse P.J., Sachs G.A. (2006) Speaking of research advance directives: planning for future research participation. Neurology. 66, 1361-1366.

Sugarman J., Cain C., Wallace R., Welsh-Bohmer K.A. (2001) How proxies make decisions about research for patients with Alzheimer's disease. J Am Geriatr Soc. 49, $1110-1119$.

Sulmasy D.P., Snyder L. (2010) Substituted interests and best judgments: an integrated model of surrogate decision making. JAMA. 304, 1946-1947.

Wendler D., Prasad K. (2001) Core safeguards for clinical research with adults who are unable to consent. Ann Intern Med. 135, 514-523.

World Medical Association (1964) Declaration of Helsinki. World Medical Association, Ferney-Voltaire. 